\title{
Fabrication of lanthanum-doped thorium dioxide by high-energy ball milling and spark plasma sintering
}

\author{
Spencer M. Scott,,${ }^{1}$ Tiankai Yao, ${ }^{1}$ Fengyuan Lu, ${ }^{2}$ Guoqing Xin, ${ }^{1}$ Weiguang Zhu, ${ }^{1}$ Jie Lian ${ }^{1, *}$ \\ ${ }^{1}$ Department of Mechanical, Aerospace, and Nuclear Engineering, Rensselaer Polytechnic \\ Institute, $1108^{\text {th }}$ Street, Troy, NY 12180, USA \\ ${ }^{2}$ Department of Mechanical \& Industrial Engineering, Louisiana State University, Baton Rouge, \\ LA 70803, USA
}

*Corresponding Author. Tel: 518 276-6081. Email: lianj@rpi.edu

\begin{abstract}
High-energy ball milling was used to synthesize $\mathrm{Th}_{1-\mathrm{x}} \mathrm{La}_{\mathrm{x}} \mathrm{O}_{2-0.5 \mathrm{x}}(\mathrm{x}=0.09,0.23)$ solid solutions, as well as improve the sinterability of $\mathrm{ThO}_{2}$ powders. Dense La-doped $\mathrm{ThO}_{2}$ pellets with theoretical density above $94 \%$ were consolidated by spark plasma sintering at temperatures above $1400{ }^{\circ} \mathrm{C}$ for 20 mins, and the densification behavior and the non-equilibrium effects on phase and structure were investigated. A lattice contraction of the SPS-densified pellets occurred with increasing ball milling duration, and a secondary phase with increased La-content was observed in La-doped pellets. A dependence on the La-content and sintering duration for the onset of localized phase segregation has been proposed. The effects of high-energy ball milling, La-content, and phase formation on the thermal diffusivity were also studied for La-doped $\mathrm{ThO}_{2}$ pellets by laser flash measurement. Increasing La-content and high energy ball milling time decreases thermal diffusivity; while the sintering peak temperature and holding time beyond $1600{ }^{\circ} \mathrm{C}$ dramatically altered the temperature dependence of the thermal diffusivity beyond 600 ${ }^{\circ} \mathrm{C}$.
\end{abstract}




\section{Introduction:}

Thorium has drawn the attention of the nuclear community due to the potential implications of thorium fuel cycle, particularly on potentially-improved operational safety and nonproliferation. The thorium fuel cycle is based upon the fertile nature of $\mathrm{Th}^{232}$, the sole isotope existing in a significant quantity in nature, and its transmutation to fissile $\mathrm{U}^{233}$. The major benefits of the thorium fuel cycle are derived from the material properties of thorium, including the high melting point of $\mathrm{ThO}_{2}\left(3,390{ }^{\circ} \mathrm{C}\right)$ as compared to that of uranium dioxide $\left(2,865{ }^{\circ} \mathrm{C}\right)$, as well as increased thermal conductivity in $\mathrm{ThO}_{2}$ over $\mathrm{UO}_{2}$ and its lower thermal expansion [1, 2]. The oxidation behavior of thorium as a 4+ oxidation state as compared to uranium's 4+, 5+, and 6+ oxidation states benefits the fabrication and chemical stability of the fuel. The attractiveness of the thorium fuel cycle is further enhanced by the relative abundance of thorium as compared to uranium, with thorium being three to four times as common globally $[1,2]$. The thorium fuel cycle has drawn attention for being proliferation resistant due to difficulties in the acid dissolution of $\mathrm{ThO}_{2}$ as a result of the aforementioned chemical stability, and the high content of gamma-emitting $\mathrm{U}^{232}$ present in spent $\mathrm{ThO}_{2}$ fuels [1-4]. However, drawbacks associated with the thorium fuel cycle, such as the necessity of reprocessing to fully utilize the benefits of the fuel cycle, and difficulties with such reprocessing due to the aforementioned chemical stability and high gamma-emissions, have resulted in a delayed implementation of thorium technologies.

While the majority of work pertaining to $\mathrm{ThO}_{2}$ mixtures has focused on the combination of $\mathrm{ThO}_{2}$ and major actinides in mixed oxide (MOX) fuels [5-8], the incorporation of lanthanides into the $\mathrm{ThO}_{2}$ matrix has been an ongoing research topic for the nuclear community. Incorporation of lanthanides into $\mathrm{ThO}_{2}$ has been previously researched with the goal of improving the performance of the $\mathrm{ThO}_{2}$-lantainide systems for applications primarily related to 
nuclear fuels. Prior investigations have focused on the effects of lanthanide dopants on the chemical stability [9], thermal conductivity [10, 11], thermal expansion [12], lattice parameter [13], and densification [14] of $\mathrm{ThO}_{2}$-lanthanide systems.

In this study, the incorporation of the lanthanide into the $\mathrm{ThO}_{2}$ systems was achieved by a low temperature solid-state reaction by high energy ball milling (HEBM). HEBM is a room temperature solid-state synthesis technique which allows for the simultaneous reduction of particle size and facilitation of phase transformations and/or incorporation of additives. HEBM has been previously utilized for the synthesis of nuclear fuels [15], photocatalysts [16], fuel cells [17], and nuclear waste forms [18]. HEBM was used in this study as the primary means of incorporating dopants into the $\mathrm{ThO}_{2}$ matrix, and the additional effect of mechanically reducing the particle size allowed for a simple variation in starting particle sizes.

The dense La-doped $\mathrm{ThO}_{2}$ pellets were consolidated by spark plasma sintering (SPS). SPS is a field-assisted sintering technique (FAST) which enables the reduction in the temperature and hold times required for densification; this creates the potential to fabricate fully-densified ceramics with significant reductions of energy cost as compared to conventional techniques [19, 20]. SPS, as a rapid consolidation technology, has attracted extensive attention in nuclear communities for fabricating advanced nuclear fuels. For example, SPS has been utilized in sintering high-density $\mathrm{ThO}_{2}[21], \mathrm{UO}_{2}$ [22], and $\mathrm{CeO}_{2}$ [23].

This work outlines the procedure to synthesize nano-sized $\mathrm{ThO}_{2}$ powders, both with and without incorporating lanthanum oxide, through HEBM as well as the sintering routine to produce fully densified $\mathrm{ThO}_{2}$ and $\mathrm{Th}_{1-\mathrm{x}} \mathrm{La}_{\mathrm{x}} \mathrm{O}_{2-0.5 \mathrm{x}}$ pellets via SPS. SPS was used to not only densify the $\mathrm{ThO}_{2}$ and $\mathrm{Th}_{1-\mathrm{x}} \mathrm{La}_{\mathrm{x}} \mathrm{O}_{2-0.5 \mathrm{x}}$ powders, but investigate the effect of non-equilibrium conditions during sintering of doped samples. The effects of the dopant content, milling time, 
and sintering parameters on $\mathrm{ThO}_{2}$ sinterability and pellet densification were investigated. The structure and phase evolution including the phase formation and the secondary phase segregation due to non-equilibrium conditions during synthesis and sintering, and their effects on thermalphysics properties were also investigated.

\section{Experimental details}

\subsection{La incorporation and $\mathrm{ThO}_{2}$ powder fabrication}

Nano-sized powders of La-doped $\mathrm{ThO}_{2}$ were fabricated via HEBM (Fritsch, Pulverisette 7 Premium Line, Idar-Oberstein, Germany). Thorium dioxide (99.9\% purity; International BioAnalytical Industries Inc.) and lanthanum oxide (99.99\% purity; Acros Organics) powders were used without further purification. Powders were mixed at weight ratios corresponding to the desired atomic ratios, and $10 \mathrm{~g}$ of mixture was loaded into the milling jars in $10 \mathrm{~mL}$ of ethanol. HEBM was carried out using a zirconia bowl $(80 \mathrm{ml})$ and balls $(1.5 \mathrm{~mm}$ diameter $)$ for $12 \mathrm{~h}$ to 18 $\mathrm{h}$ milling time at $600 \mathrm{rpm}$, with a milling pattern of 15 minutes on, 5 minutes off, to prevent overheating of the ball mill, until the mixture was determined to be homogeneously blended by X-ray powder diffraction.

\subsection{Spark plasma sintering}

Spark plasma sintering was carried out using a Dr. Sinter ${ }^{\circledR}$ SPS-211 Lx system with a procedure similar to our previous work. [15] To prepare each sample, approximately $1 \mathrm{~g}$ of powder was loaded into a graphite die, $10 \mathrm{~mm}$ in diameter. Graphite paper $(0.20 \mathrm{~mm}$ thick $)$ was used to envelope the powders, ensuring proper fit during assembly, ease of sample retrieval after sintering, and sufficient contact to facilitate the current passing through the assembly. The die was then wrapped in a graphite felt to insulate the die from radiative heat loss during fabrication. 
The fully assembled graphite die was then arranged between graphite spacers, and loaded into the SPS between the rams of the hydraulic press with an applied pressure of $10 \mathrm{MPa}$.

Sintering was conducted under flowing argon, with current applied in a $25 \mathrm{~ms}$ on, $3 \mathrm{~ms}$ off pulse pattern. The sample temperature, which was measured using a pyrometer, was gradually increased from $25{ }^{\circ} \mathrm{C}$ to $600{ }^{\circ} \mathrm{C}$ before being increased to the peak temperature (between $1300{ }^{\circ} \mathrm{C}$ and $1700{ }^{\circ} \mathrm{C}$ ) at a rate of $100{ }^{\circ} \mathrm{C} / \mathrm{min}$ (Fig. 1). The uniaxial pressure applied by the hydraulic press was increased to $40 \mathrm{MPa}$ at a rate which allowed it to reach its maximum value simultaneously with the temperature. The peak temperature was held at for a set period of time, (5 minutes unless otherwise noted) and then cooled to $100{ }^{\circ} \mathrm{C}$ below the peak temperature at a rate of $20{ }^{\circ} \mathrm{C} / \mathrm{min}$. The applied pressure was reduced during this cooling to $10 \mathrm{MPa}$ at a rate of 6 $\mathrm{MPa} / \mathrm{min}$. The sample was then held at this reduced temperature for another 5 minutes, before being allowed to cool freely under the flowing argon.

\subsection{Characterization}

Density measurements were carried out using the Archimedes' method, by immersing the samples in deionized water using an Adam analytical scale (Danbury, NY, USA). X-ray diffraction measurements were carried out using a Panalytical X'Pert Pro system (Westborough, MA, USA) with a copper target $\left(\mathrm{K}_{\mathrm{a}}=0.15406 \mathrm{~nm}\right)$ and a step size of $\left.0.0131^{\circ}\right)$. Scanning electron microscopy (SEM) as well as energy dispersive spectroscopy (EDS) measurements were conducted using a Zeiss 1540XB CrossBeam (Jena, Germany). SEM was also conducted on a Carl Zeiss Supra (Jena, Germany). In order to reduce charging during SEM, samples were coated in a thin layer of platinum under an argon environment via a Denton Sputter system (Denton Vacuum, LLC, NJ, USA). Thermal diffusivity measurements were taken using laser flash analysis (LFA-457, NETZCH, Germany), and the samples were coated with a thin layer of 
graphite in order to promote photon absorption and consistency. Measurements were taken in an argon environment with a flow rate of $150 \mathrm{ml} / \mathrm{min}$. Sample density was corrected to $100 \%$ theoretical density in order to correct for density variation between samples of identical compositions.

\section{Results and discussion}

X-ray powder diffraction of the $\mathrm{HEBM}$ mixture of $\mathrm{ThO}_{2}$ and $\mathrm{La}_{2} \mathrm{O}_{3}$ indicates that the incorporation of $\mathrm{La}_{2} \mathrm{O}_{3}$ into the $\mathrm{ThO}_{2}$ matrix occurs, with the patterns matching that of $\mathrm{ThO}_{2}$, with no observable residue peaks and minor peak shifting identified from pure $\mathrm{ThO}_{2}$ without ball milling (Fig. 2). This agrees with prior studies regarding the $\mathrm{ThO}_{2}-\mathrm{La}_{2} \mathrm{O}_{3}$ system, which demonstrated the formation of a solid solution, with La solubility up to 50 mol.\% via coprecipitation [14]. Minor lattice parameter changes were observed among the powders, with $12 \mathrm{~h}$ HEBM resulting in a minor $(0.0001 \mathrm{~nm})$ decrease in lattice parameter and La-contents of 0.09 and 0.23 resulted in increases of $0.0003 \mathrm{~nm}$ and $0.0008 \mathrm{~nm}$, respectively, as compared to $\mathrm{ThO}_{2}$ undergoing similar HEBM. The trend of increasing lattice parameter with La incorporation agrees with prior research, as the ion size mismatch between $\mathrm{Th}^{+4}(0.105 \mathrm{~nm})$ and $\mathrm{La}^{+3}(0.116$ $\mathrm{nm}$ ) should result in an expanded lattice with the replacement of Th with La. However, the magnitude of lattice expansion is reduced from prior experiments, suggesting that lattice contractions from HEBM play a competing role. Morphological measurements by SEM images display micron-sized agglomerates, a typical feature of high energy ball. Bright-field TEM images (Fig. 3) show ultrafine nanoparticles with the agglomerates with the particle size in the range of 10-20 nm for both $\mathrm{ThO}_{2}$ and La-doped $\mathrm{ThO}_{2}$ upon HEBM, significantly improving the sinterability of the ball milled powders as compared with pristine micro-sized powders. 
SPS densification of $\mathrm{ThO}_{2}$ powders (Fig. 4A) indicates an improvement in sinterability with increasing mill time, with a general trend of density increasing with peak sintering temperature and then saturating as density approached the theoretical maximum. The enhancement of sinterability is exemplified by the $300{ }^{\circ} \mathrm{C}$ reduction in peak sintering temperature required to achieve full densification ( $\geq 95 \%$ T.D.) between the as-purchased $\mathrm{ThO}_{2}$ and the $18 \mathrm{~h}$ HEBMed$\mathrm{ThO}_{2}$. This improvement in sinterability may be attributed to increasing particle surface area with milling time. Similar behavior has been previously reported in literature, with the reduction in starting $\mathrm{ThO}_{2}$ particle size reducing the temperature threshold for the onset of sintering, resulting in increased density under identical sintering conditions [21].

SPS densification of $\mathrm{Th}_{1-\mathrm{x}} \mathrm{La}_{\mathrm{x}} \mathrm{O}_{2-0.5 \mathrm{x}}$ powders (Fig. 4B) resulted in a similar trend between density and peak sintering temperature, with density increasing with temperature. However, the La-doped powders were noticeably less densified under similar conditions, even after correcting for the lower theoretical density. The La-doped powder displayed sintering properties closer to those of the as-purchased $\mathrm{ThO}_{2}$, as compared to the powders processed using HEBM, despite having been synthesized via $12 \mathrm{~h}$ of HEBM. This is not consistent with the expectation in which the incorporation of $\mathrm{La}$ into the $\mathrm{ThO}_{2}$ system increases the concentration of the oxygen vacancies, promoting atomic diffusion and thus sintering processing. This reduction in sinterability for La-doped $\mathrm{ThO}_{2}$ may be due to the structural distortion upon La incorporation.

SEM images (Fig. 5) indicate that increased milling time resulted in minor reductions in grain sizes of $\mathrm{ThO}_{2}$ pellets, with samples sintered at $1600{ }^{\circ} \mathrm{C}$ and undergoing HEBM for $12 \mathrm{~h}$ and 18h displayed grain sizes of $3.2 \pm 0.6 \mu \mathrm{m}$ and $2.9 \pm 1.1 \mu \mathrm{m}$, respectively. This is consistent with the grain refinement of the starting materials for sintering by elongated ball milling. SEM images (Figs. 5C and 5D) further suggest La-incorporation into $\mathrm{ThO}_{2}$ reduces grain growth during 
sintering, with $\mathrm{Th}_{0.91} \mathrm{La}_{0.09} \mathrm{O}_{1.955}$ and $\mathrm{Th}_{0.77} \mathrm{La}_{0.23} \mathrm{O}_{1.885}$ pellets sintered at $1600{ }^{\circ} \mathrm{C}$ displayed grain sizes of $1.4 \pm 0.4 \mu \mathrm{m}$ and $1.0 \pm 0.2 \mu \mathrm{m}$, respectively. This reduction in grain size is presumably due to a reduction in atomic diffusion during sintering due to La-incorporation combined with a pinning of the grain boundaries by La-rich phases. Little-to-no grain coarsening was observed in La-doped samples when held at peak temperature during sintering for an hour, with $\mathrm{Th}_{0.91} \mathrm{La}_{0.09} \mathrm{O}_{1.955}$ pellets sintered at $1600{ }^{\circ} \mathrm{C}$ for 1 hour displayed grain sizes of $1.5 \pm 0.3 \mu \mathrm{m}$. This was an unexpected phenomenon and may associated with the aforementioned reductions in grain sizes observed with La-incorporation as compared to pure $\mathrm{ThO}_{2}$ pellets. SEM images further displayed the low porosity of the sintered pellets, reinforcing the high degree of densification measured using Archimedes' method.

XRD results (Fig. 6 and 7) of $\mathrm{HEBM}-\mathrm{ThO}_{2}$ and La-doped $\mathrm{ThO}_{2}$ samples sintered at different temperatures by SPS clearly show lattice contraction upon HEBM and lattice expansion with the incorporation of $\mathrm{La}_{2} \mathrm{O}_{3}$ as evidenced by the XRD diffraction peak shifting. The decrease in lattice parameter due to HEBM of the starting powders is readily apparent by comparing the sintered $\mathrm{ThO}_{2}$ samples (Fig. 7A). HEBM resulted in a reduction of approximately $0.0015 \mathrm{~nm}$ for the densified pellets, with minor differences between $12 \mathrm{~h}$ and $18 \mathrm{~h}$ milling times. The minor changes in lattice parameter between milling times, coupled with the increased sinterability further supports the idea that the lattice parameter changes are due to a plastic deformation and strain induced on the lattice during HEBM; while the enhancement to sinterability may be associated with reductions in particle size.

XRD peak positions and lattice parameters prior to $1600{ }^{\circ} \mathrm{C}$ peak sintering temperature display slight changes from $\mathrm{ThO}_{2}$ undergoing identical HEBM and SPS, with $\mathrm{Th}_{0.91} \mathrm{La}_{0.09} \mathrm{O}_{1.955}$ and $\mathrm{Th}_{0.77} \mathrm{La}_{0.23} \mathrm{O}_{1.885}$ displaying $0.0002 \mathrm{~nm}$ and $0.0009 \mathrm{~nm}$ increase in lattice parameter from 
12h $\mathrm{HEBM} \mathrm{ThO}_{2}$ at $1500{ }^{\circ} \mathrm{C}$, respectively (Fig. 8). The lattice expansion upon La incorporation is due to the difference in ion size between $\mathrm{Th}^{+4}(0.105 \mathrm{~nm})$ and $\mathrm{La}^{+3}(0.116 \mathrm{~nm})$ [24]. The resulting structural distortion observed from the incorporation of $\mathrm{La}$ into the $\mathrm{ThO}_{2}$ matrix [11] is consistent with previous results of reducing sintering in $\mathrm{Y}_{2} \mathrm{O}_{3}$-doped $\mathrm{CeO}_{2}$ with increasing dopant concentration [25].

A minor peak associated with $\mathrm{La}_{2} \mathrm{O}_{3}$ was observed at $1600{ }^{\circ} \mathrm{C}$ in $\mathrm{Th}_{0.91} \mathrm{La}_{0.09} \mathrm{O}_{1.955}$ and at and beyond $1600{ }^{\circ} \mathrm{C}$ in $\mathrm{Th}_{0.77} \mathrm{La}_{0.23} \mathrm{O}_{1.885}$. The existence of the minor $\mathrm{La}_{2} \mathrm{O}_{3}$ phase may be caused by an incomplete incorporation of $\mathrm{La}_{2} \mathrm{O}_{3}$ into $\mathrm{ThO}_{2}$ at room temperature by $\mathrm{HEBM}$ and rapid consolidation of SPS at temperature below $1600{ }^{\circ} \mathrm{C}$. The unreacted $\mathrm{La}_{2} \mathrm{O}_{3}$ becomes increasingly more crystalline as temperature increases and observable via XRD. The other possibility might be localized incorporation of $\mathrm{La}_{2} \mathrm{O}_{3}$ into $\mathrm{ThO}_{2}$ above its solubility limit during $\mathrm{HEBM}$ and the gradual segregation upon SPS sintering. However, the solubility of La reaches to $\mathrm{x}=0.50$ for $\mathrm{La}_{\mathrm{x}} \mathrm{Th}_{1-\mathrm{x}} \mathrm{O}_{2-0.5 \mathrm{x}}$ prepared by a co-precipitation approach. A decrease in $\mathrm{La}_{2} \mathrm{O}_{3}$ solubility was reported when incorporated using solid-state reactions, with a maximum solubility observed for a La-content of $\mathrm{x}=0.30$, as compared to formation using techniques such as co-precipitation [11]. No potential explanation was offered as to the solubility decrement between solid-state reactions and co-precipitation. Therefore, it is unlikely that the emergence of the minor phase $\mathrm{La}_{2} \mathrm{O}_{3}$ was caused by localized incorporation above its solubility in $\mathrm{ThO}_{2}$ for $\mathrm{Th}_{0.91} \mathrm{La}_{0.09} \mathrm{O}_{1.955}$ since the incorporation amount is significantly less than the solubility observed for solid-state reaction. Because the $\mathrm{Th}_{0.91} \mathrm{La}_{0.09} \mathrm{O}_{1.955}$ samples sintered at $1700{ }^{\circ} \mathrm{C}$ or elongated sintering at $1600{ }^{\circ} \mathrm{C}$ for $1 \mathrm{~h}$ did not present $\mathrm{La}_{2} \mathrm{O}_{3}$, it implies that $\mathrm{La}_{2} \mathrm{O}_{3}$ may not be completely incorporated into $\mathrm{ThO}_{2}$ upon HEBM at room temperature and rapid consolidation of SPS at lower sintering temperature. At $1600{ }^{\circ} \mathrm{C}$, unincorporated and pre-damaged $\mathrm{La}_{2} \mathrm{O}_{3}$ by HEBM displays increased crystallinity, 
responsible for the observation of the minor phase in XRD. Unreacted $\mathrm{La}_{2} \mathrm{O}_{3}$ was completely incorporated into the $\mathrm{ThO}_{2}$ matrix with the increase of sintering temperature and elongated sintering time approaching to equilibrium conditions. For $\mathrm{Th}_{0.77} \mathrm{La}_{0.23} \mathrm{O}_{1.885}$, due to the higher amount of La incorporation, the minor phase still existed for SPS densified pellets at $1700{ }^{\circ} \mathrm{C}$ and $1600{ }^{\circ} \mathrm{C}$ for $1 \mathrm{~h}$.

A temperature dependent phase evolution also occurs at and above $1600{ }^{\circ} \mathrm{C}$ during SPS. Based on the peak positions (Fig. 7), major phases present at $1600{ }^{\circ} \mathrm{C}$ in La-doped samples appear to correspond with La-doped $\mathrm{ThO}_{2}$ of varying La content, La-rich at lower angle, and Ladeficient at higher angle, along with the emergence of minor peaks associated with $\mathrm{La}_{2} \mathrm{O}_{3}$. The emergence of the lower angle peak, associated with a La-doped $\mathrm{ThO}_{2}$ phase of higher content (La-rich), as well as minor peaks associated with $\mathrm{La}_{2} \mathrm{O}_{3}$ with increasing sintering temperature is readily observed through the change in $\mathrm{Th}_{0.77} \mathrm{La}_{0.23} \mathrm{O}_{1.885}$ samples (Fig. 8B). At $1500{ }^{\circ} \mathrm{C}$. XRD indicates a single phase, matching the pattern of $\mathrm{ThO}_{2}$, with a $0.0009 \mathrm{~nm}$ increase in lattice parameter compared to samples prepared via $12 \mathrm{~h}$ HEBM. This minor change is a result of the competition between lattice contraction due to HEBM and the lattice expansion from La incorporation. At $1600{ }^{\circ} \mathrm{C}$, the intensity of the $\mathrm{ThO}_{2}$ peak is dramatically reduced, along with the emergence of the La-rich phase, accompanied by minor peaks indicating $\mathrm{La}_{2} \mathrm{O}_{3}$. At $1700{ }^{\circ} \mathrm{C}$, the peaks associated with the La-deficient phase are completely eliminated, replaced by the La-rich phase and the minor $\mathrm{La}_{2} \mathrm{O}_{3}$ peaks. This is consistent with the previous analysis that $\mathrm{La}_{2} \mathrm{O}_{3}$ was not completely incorporated into $\mathrm{ThO}_{2}$ matrix during HEBM at room temperature and SPS rapid sintering at lower temperatures, leading to a dominant La-deficient $\mathrm{ThO}_{2}$ phases due to the nonequilibrium conditions of SPS. At higher sintering temperature and elongated sintering duration, 
the unreacted $\mathrm{La}_{2} \mathrm{O}_{3}$ was gradually incorporated into the La-deficient phase, transforming to the La-enriched phase.

The non-equilibrium effects of HEBM and SPS were thought to contribute to the observed phase evolution phenomenon; in order to test this, the SPS routine was modified, extending the holding time of the peak temperature from 5 mins to $1 \mathrm{~h}$. This was carried out for both the investigated $\mathrm{La}$ contents at $1600{ }^{\circ} \mathrm{C}$. The resulting XRD patterns indicated diverging results based on La-content (Fig. 7). The $\mathrm{Th}_{0.91} \mathrm{La}_{0.09} \mathrm{O}_{1.955}$ sample produced a pattern closely resembling that of the $1500{ }^{\circ} \mathrm{C}$ and $1700{ }^{\circ} \mathrm{C}$ samples, with a singular phase similar to the $12 \mathrm{~h} \mathrm{HEBM} \mathrm{ThO}_{2}$. The $\mathrm{Th}_{0.77} \mathrm{La}_{0.23} \mathrm{O}_{1.885}$ sample produced a similar pattern to the associated $1600{ }^{\circ} \mathrm{C}$ sample, with minor $\mathrm{La}_{2} \mathrm{O}_{3}$ peaks present, along with a diminished $\mathrm{ThO}_{2}$ peak and more intense La-doped $\mathrm{ThO}_{2}$ peaks. This suggests that the non-equilibrium effects of SPS may play a role in the observed phase evolution; however the dopant content significantly impacts the degree of reincorporation into the strained $\mathrm{ThO}_{2}$ matrix, as the availability of La changes throughout the material. This may be supported by the previously discussed decrease in $\mathrm{La}_{2} \mathrm{O}_{3}$ solubility when incorporated into $\mathrm{ThO}_{2}$ via solid-state reactions [11].

The calculated lattice parameters of the La-rich phases based on lattice parameter changes from prior studies [9, 13], when corrected for the lattice contraction induced via HEBM, correspond to a La content of $\mathrm{x} \approx 0.27$ for $\mathrm{Th}_{0.91} \mathrm{La}_{0.91} \mathrm{O}_{1.955}$ and $\mathrm{x} \approx 0.33$ for $\mathrm{Th}_{0.77} \mathrm{La}_{0.23} \mathrm{O}_{1.885}$, near the solubility limit experimentally determined for solid-state reactions. The La-content of the Larich phase corresponding to the solubility limit, along with the presence of minor $\mathrm{La}_{2} \mathrm{O}_{3}$ peaks suggest that during the non-equilibria sintering conditions of SPS, non-uniform and localized incorporation of $\mathrm{La}_{2} \mathrm{O}_{3}$ in $\mathrm{ThO}_{2}$ occurs, leaving some unreacted $\mathrm{La}_{2} \mathrm{O}_{3}$ in the $\mathrm{ThO}_{2}-\mathrm{La}_{2} \mathrm{O}_{3}$ mixture. This resulted in a La-deficient phase closely resembling $\mathrm{ThO}_{2}$ for the pellets sintered 
below $1600{ }^{\circ} \mathrm{C}$ and a La-enriched $\mathrm{ThO}_{2}$ and excess $\mathrm{La}_{2} \mathrm{O}_{3}$ at $1600{ }^{\circ} \mathrm{C}$. Upon sufficient sintering temperature or duration, as observed in the $\mathrm{Th}_{0.91} \mathrm{La}_{0.09} \mathrm{O}_{1.955}$ samples sintered at $1700{ }^{\circ} \mathrm{C}$ and $1600{ }^{\circ} \mathrm{C}$ for 1 hour, the incorporation of $\mathrm{La}_{2} \mathrm{O}_{3}$ becomes more uniform, leading to the elimination of minor $\mathrm{La}_{2} \mathrm{O}_{3}$ and the La-rich phase and a singular La-doped phases corresponding to the designed composition of $\mathrm{Th}_{0.91} \mathrm{La}_{0.09} \mathrm{O}_{1.955}$. On the other hand, for the $\mathrm{Th}_{0.77} \mathrm{La}_{0.23} \mathrm{O}_{1.885}$ samples, due to the higher La content, limited incorporation of $\mathrm{La}$ into $\mathrm{ThO}_{2}$ occurs at $1500{ }^{\circ} \mathrm{C}$, but the La-deficient peaks were eliminated in the samples sintered at $1700{ }^{\circ} \mathrm{C}$, suggesting the increased temperature enabled the incorporation of La to the solubility limit for solid-state reaction in $\mathrm{ThO}_{2}-\mathrm{La}_{2} \mathrm{O}_{3}$.

The phase decomposition and elemental partitioning into La-rich and La-depleted $\mathrm{ThO}_{2}$ phases were further confirmed by SEM images and energy dispersive spectroscopy (EDS). Backscattered electron images of the $\mathrm{Th}_{0.77} \mathrm{a}_{0.23} \mathrm{O}_{1.885}$ sample sintered at $1600{ }^{\circ} \mathrm{C}$ (Fig. 9A) indicate the presence of a secondary phase of lower atomic mass at the sub-micron scale, distributed bimodally between nano-metered (less than $50 \mathrm{~nm}$ ) and sub-micron (200-500 nm) sizes. This darker secondary phase was confirmed via EDS (Figs. 9B, 9C) to be a La-rich phase with the surrounding lighter phase displaying La-deficiency. The confirmation of the La-rich secondary phase occurring at the sub-micron scale reinforces the idea that localized phase segregation occurred due to the sintering conditions and La-incorporation.

Thermal diffusivity measurements indicate a strong decrease in diffusivity among $\mathrm{ThO}_{2}$ samples prepared using HEBM (Fig. 10A), with increasing mill time resulting in a minor degradation of diffusivity. This is most likely a result of an increase in the number of grain boundaries due to reduced grain size; because the grain boundaries act as phonon scattering sites, this results in a decreased thermal diffusivity in the samples prepared using HEBM. This 
dependence of thermal performance on grain size has been previously observed in yttriastabilized zirconia [26].

$\mathrm{La}_{2} \mathrm{O}_{3}$ incorporation into $\mathrm{ThO}_{2}$ dramatically decreased the thermal diffusivity of samples, beyond the decrease associated with HEBM processing (Figs. 10B, 10C), with increasing La content resulting in a further decrease in thermal diffusivity. This reduction in thermal diffusivity was expected, and agrees with prior studies on the thermal effects of $\mathrm{La}$ doping on $\mathrm{ThO}_{2}[10,11$, 13]. The general trend of thermal diffusivity for La-doped samples at lower SPS temperatures $\left(\leq 1600{ }^{\circ} \mathrm{C}\right)$ was a gradual decrease of thermal diffusivity with temperature until approximately $600{ }^{\circ} \mathrm{C}$, followed by a gradual increase in diffusivity as temperature increased, with the diffusivity at $1000{ }^{\circ} \mathrm{C}$ nearly reaching the room temperature diffusivity of the sample. The diffusivity reduction in the La-doped samples as compared to $\mathrm{ThO}_{2}$ with identical $\mathrm{HEBM}$ processing is considerably less pronounced at higher temperatures, and may be further reduced at temperatures above $1000{ }^{\circ} \mathrm{C}$ based on the observed trends. La-doped samples sintered above $1600{ }^{\circ} \mathrm{C}$ and those allowed to reach equilibrium during sintering (held at peak temperature for 1 hour), displayed different thermal diffusivity trends than identically processed samples that underwent the standard 5 minutes hold at peak SPS temperature at or below $1600{ }^{\circ} \mathrm{C}$ (Figs. 10B, 10C). These samples exhibited a similar trend to pure $\mathrm{ThO}_{2}$, a steady decrease in thermal diffusivity with temperature while still displaying the reduction in diffusivity associated with La doping.

A transition from photon-dominated heat transport to electron-dominate transport occurred in $\mathrm{UO}_{2}$, leading to the increase of thermal conductivity above $1500{ }^{\circ} \mathrm{C}$. However, similar to $\mathrm{UO}_{2}$, $\mathrm{ThO}_{2}$ is a wide band gap material, and electron contribution may not dominate thermal transport above $600{ }^{\circ} \mathrm{C}$. This change in the temperature dependence of the thermal diffusivity may be 
attributed to competition between phase/microstructure evolution and strain relaxation of the samples upon different sintering or measurement conditions that impact the phonon transport and interaction behavior. Specifically, the defect resistivity increases as a result of micro-strain and defect accumulation and heteroatom scattering upon the large sized $\mathrm{La}^{3+}$ incorporation into $\mathrm{Th}^{4+}$ sub-lattices and creation of oxygen vacancies[10]. The existence of the La-enriched and Thenriched dual phases in the La-incorporated samples upon rapid SPS consolidation also increases the defect thermal resistivity due to interfacial and boundary scattering. The increased thermal diffusivity occurred above $600{ }^{\circ} \mathrm{C}$ for the La-incorporated samples maybe attributed to strain relaxation upon higher temperature annealing. In addition, nano-sized secondary phase may be incorporated into the Th-enriched phase during laser flash measurement, reducing the interfacial resistivity. On the other hand, higher temperature sintering $\left(1700{ }^{\circ} \mathrm{C}\right)$ and/or prolonged holding time $(1 \mathrm{hr})$ at peak sintering temperature allow more uniform incorporation of La in the $\mathrm{ThO}_{2}$ phases and thus dominate phonon scattering resulting from heterogeneous atom doping. This may result in thermal behavior similar to that of pure $\mathrm{ThO}_{2}$ samples, in which the phonon scattering dominates the thermal transport at measurement temperatures up to $1000{ }^{\circ} \mathrm{C}$, but with the thermal diffusivity penalty expected for $\mathrm{La}_{2} \mathrm{O}_{3}$ incorporation.

\section{Conclusions}

High-energy ball milling was shown to improve sinterability in $\mathrm{ThO}_{2}$ powders while being a suitable method for the incorporation of $\mathrm{La}_{2} \mathrm{O}_{3}$ into $\mathrm{ThO}_{2}$. Spark plasma sintering densification was used to achieve high-density pellets of $\mathrm{ThO}_{2}$ and $\mathrm{Th}_{1-\mathrm{x}} \mathrm{La}_{\mathrm{x}} \mathrm{O}_{2-0.5 \mathrm{x}}$. A complete incorporation of lanthanum was not achieved by solid state reaction at room temperature by HEBM. Temperature dependent phase segregation of $\mathrm{La}_{2} \mathrm{O}_{3}$ and La-rich and La-deficient $\mathrm{Th}_{1-\mathrm{x}} \mathrm{La}_{\mathrm{x}} \mathrm{O}_{2-0.5 \mathrm{x}}$ was observed via X-ray diffraction, and supported by thermal diffusivity measurements. The 
non-equilibrium conditions of SPS were shown to affect phase segregation based on the dopant content, with the lower dopant content maintaining a single phase, and the higher content displaying the phase segregation, potentially due to the previously observed decrease in $\mathrm{La}_{2} \mathrm{O}_{3}$ solubility associated with the solid-state reaction synthesis of $\mathrm{Th}_{1-\mathrm{x}} \mathrm{La}_{\mathrm{x}} \mathrm{O}_{2-0.5 \mathrm{x}}$. The phase behavior of the La-doped $\mathrm{ThO}_{2}$ is correlated with the amount of La doping and incorporation of lanthanum into $\mathrm{ThO}_{2}$ affected by SPS temperature and duration. $\mathrm{La}_{2} \mathrm{O}_{3}$ incorporation into $\mathrm{ThO}_{2}$ was shown to dramatically reduce thermal diffusivity, with the studied non-equilibria effects and associated phase segregation demonstrably altering the temperature dependence of thermal diffusivity.

\section{Acknowledgements}

This work was financially supported by a NSF career award DMR 1151028. Thermal measurement of the SPS-densified $\mathrm{ThO}_{2}$ pellets upon La incorporation was supported by the DOE Nuclear Energy University Program (NEUP) under the award of DE-NE0008440. 


\section{Graphical Abstract:}

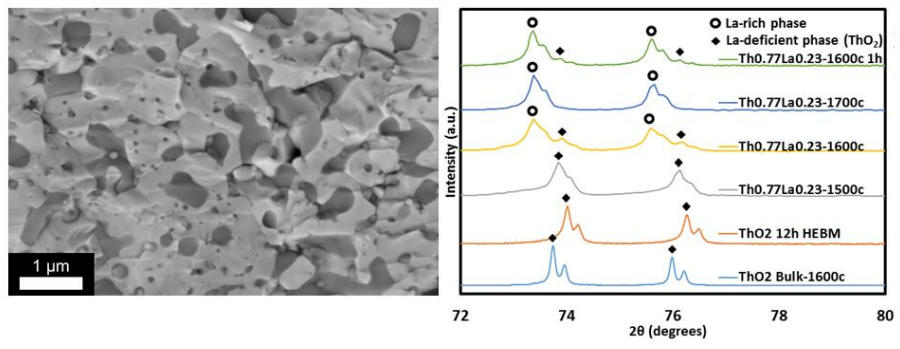

Highlights:

- Lanthanum incorporation into $\mathrm{ThO}_{2}$ by high energy ball milling and rapid consolidation of densified pellets by spark plasma sintering

- Elucidation of phase behavior of the La-doped $\mathrm{ThO}_{2}$ and their correlation with the amount of La doping, incorporation of La and SPS sintering temperature/duration

- Investigation of the effects of La incorporation and high energy ball milling on the thermal transport behavior of the La-doped $\mathrm{ThO}_{2}$. 


\section{Figures:}

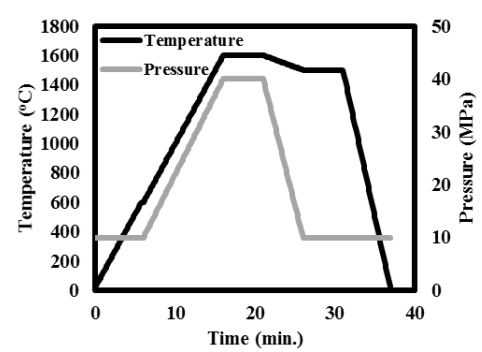

Fig. 1

Typical spark plasma sintering temperature-pressure profile used to density $\mathrm{ThO}_{2}$ powders. The maximum temperature was reached at a heating rate of $100{ }^{\mathrm{p}} \mathrm{C} / \mathrm{min}$ and maintained for 5 minutes.

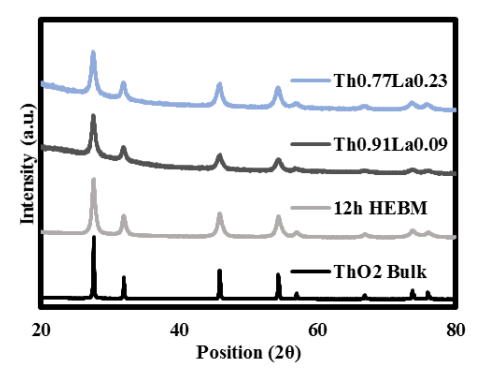

Fig. 2

X-ray powder diffraction patterns of $\mathrm{ThO}_{2}$ and $\mathrm{Th}_{1-\mathrm{x}} \mathrm{La}_{\mathrm{x}} \mathrm{O}_{2-0.5 \mathrm{x}}$ prior to sintering. The HEBM and $\mathrm{Th}_{1-\mathrm{x}} \mathrm{La}_{\mathrm{x}} \mathrm{O}_{2-0.5 \mathrm{x}}$ patterns match the commercial $\mathrm{ThO}_{2}$ pattern with minor lattice contractions. No residue peaks or secondary phases are observable in the $\mathrm{Th}_{1-\mathrm{x}} \mathrm{La}_{\mathrm{x}} \mathrm{O}_{2-0.5 \mathrm{x}}$ patterns.

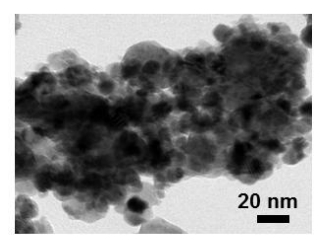

A HeBM-12h $\mathrm{ThO}_{2}$

Fig. 3

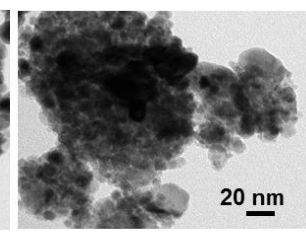

B $\mathrm{Th}_{0.91} \mathrm{La}_{0.09} \mathrm{O}_{1.955}$

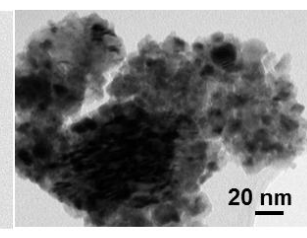

C $\mathrm{Th}_{0.77} \mathrm{La}_{0.23} \mathrm{O}_{1.885}$$$
\text { (1) }
$$ 
Bright-field TEM imaging of 12 hour HEBM: (A) $\mathrm{ThO}_{2}$ (B) $\mathrm{Th}_{0.91} \mathrm{La}_{0.09} \mathrm{O}_{1.955}$ (C) $\mathrm{Th}_{0.77} \mathrm{La}_{0.23} \mathrm{O}_{1.885}$. Particle sizes range from $10-20 \mathrm{~nm}$ for milled powders.

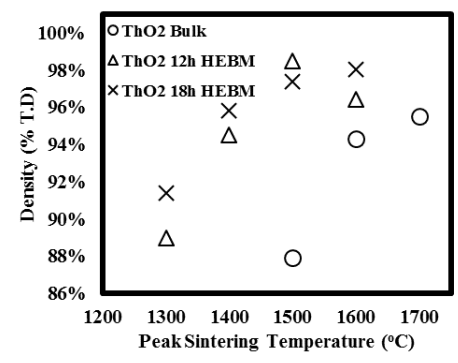

Fig. 4

Density of

(A) $\mathrm{ThO}_{2}(\mathrm{~B}) \mathrm{Th}_{1-}$

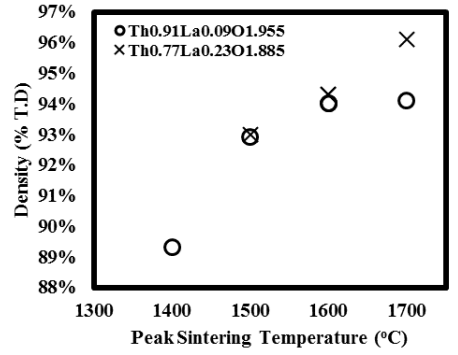

sintered pellets:

HEBM

$$
{ }_{x} \mathrm{La}_{\mathrm{x}} \mathrm{O}_{2-0.5 \mathrm{x}} .
$$

significantly

reduced the temperature required to reach full densification as compared to bulk powders. Lacontent does not appear to significantly affect the SPS densification behavior.

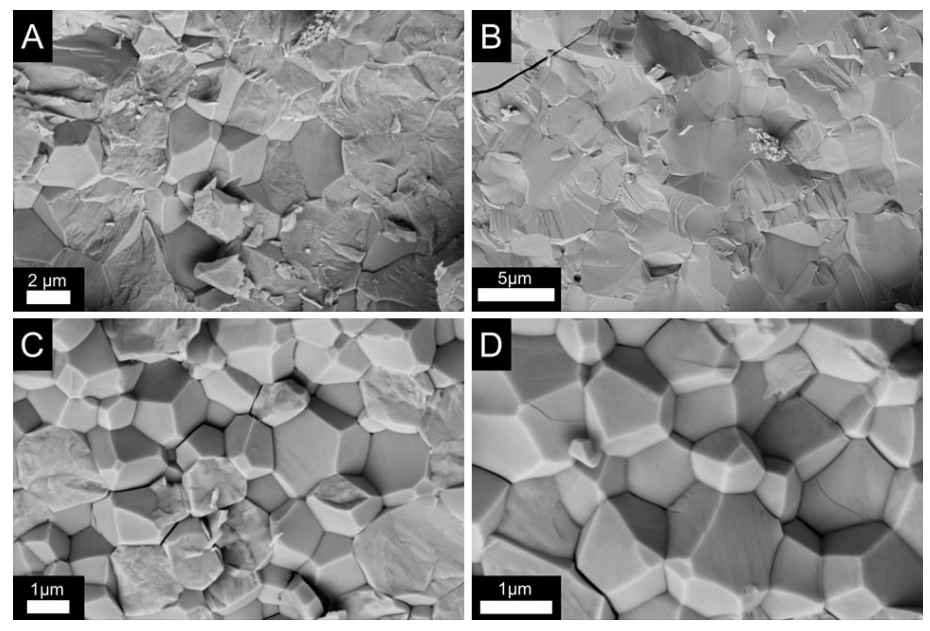

Fig. 5

SEM images of samples sintered at $1600{ }^{\circ} \mathrm{C}$ : (A) $12 \mathrm{~h} \mathrm{HEBM} \mathrm{ThO}_{2}$ (B) $18 \mathrm{~h} \mathrm{HEBM} \mathrm{ThO}_{2}$ (C) $\mathrm{Th}_{0.91} \mathrm{La}_{0.09} \mathrm{O}_{1.955}$ (D) $\mathrm{Th}_{0.91} \mathrm{La}_{0.09} \mathrm{O}_{1.955}$ held at $1600{ }^{\circ} \mathrm{C}$ for $1 \mathrm{~h}$. 

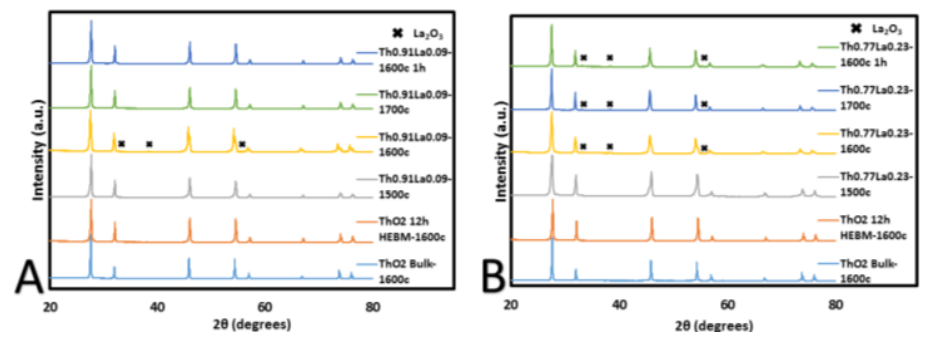

Fig. 6

X-ray diffraction results of (A) $\mathrm{Th}_{0.91} \mathrm{La}_{0.09} \mathrm{O}_{1.955}$ (B) $\mathrm{Th}_{0.77} \mathrm{La}_{0.23} \mathrm{O}_{1.885}$ pellets. The presence of minor $\mathrm{La}_{2} \mathrm{O}_{3}$ peaks are denoted.
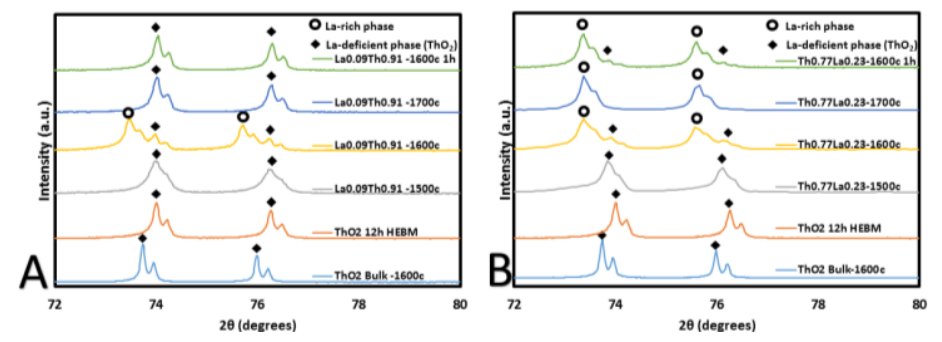

Fig. 7

X-ray diffraction results from 72 to 80 degrees $2 \theta$ of (A) $\mathrm{Th}_{0.91} \mathrm{La}_{0.09} \mathrm{O}_{1.955}(\mathrm{~B})$ $\mathrm{Th}_{0.77} \mathrm{La}_{0.23} \mathrm{O}_{1.885}$ pellets. La-rich and La-deficient $\left(\mathrm{ThO}_{2}\right)$ phase are denoted. Phase segregation is visible in both samples starting at $1600{ }^{\circ} \mathrm{C}$, followed by a singular phase, of higher angle for $\mathrm{Th}_{0.91} \mathrm{La}_{0.09} \mathrm{O}_{1.955}$ and lower angle for $\mathrm{Th}_{0.91} \mathrm{La}_{0.09} \mathrm{O}_{1.955}$, at $1700{ }^{\circ} \mathrm{C}$.
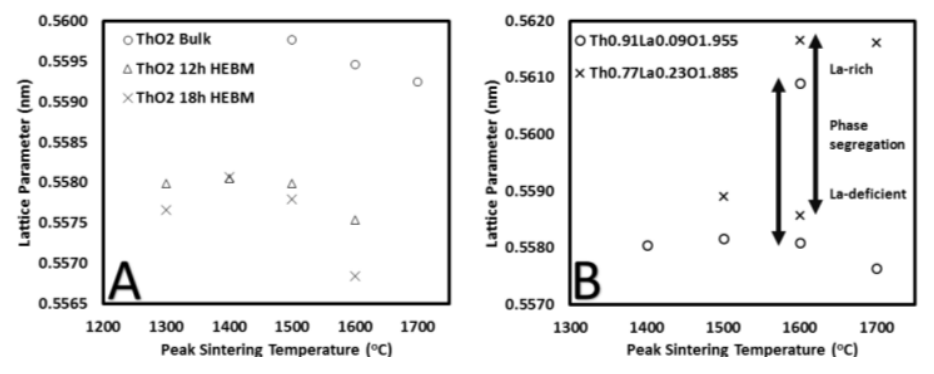

Fig. 8

Lattice parameter of sintered pellets: (A) $\mathrm{ThO}_{2}$ (B) $\mathrm{Th}_{1-\mathrm{x}} \mathrm{La}_{\mathrm{x}} \mathrm{O}_{2-0.5 \mathrm{x}}$. Minor lattice parameter changes are observed between

milling times in $\mathrm{ThO}_{2}$ samples. Phase segregation is denoted in $\mathrm{Th}_{1-\mathrm{x}} \mathrm{La}_{\mathrm{x}} \mathrm{O}_{2-0.5 \mathrm{x}}$ by arrows, showing the difference in lattice parameter between the La-rich and La-deficient phases. 


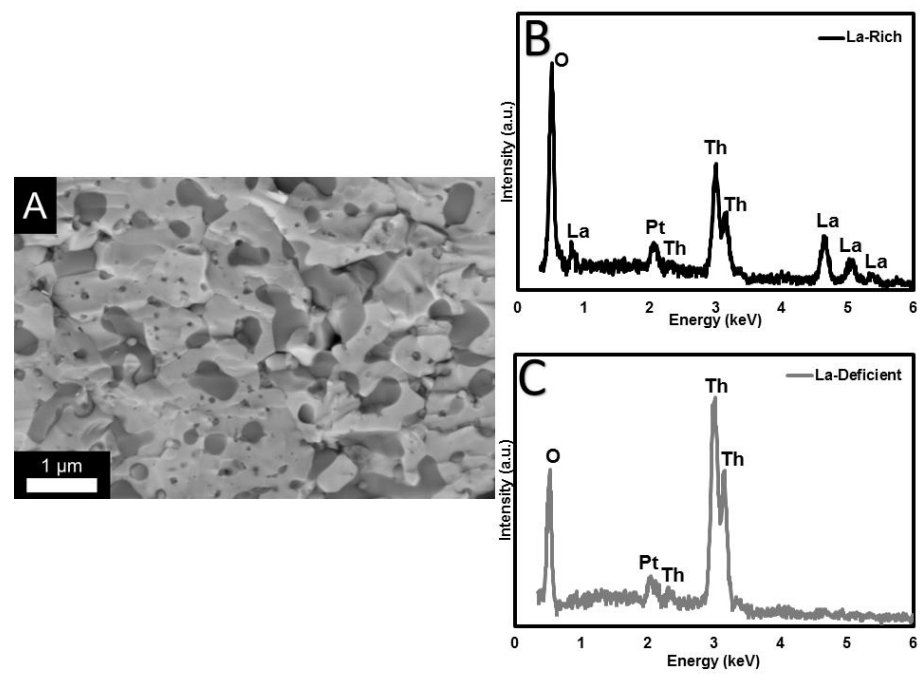

Fig. 9

Energy dispersive spectroscopy of $\mathrm{Th}_{0.77} \mathrm{La}$ ${ }_{0.23} \mathrm{O}_{1.885}$ sintered at $1600{ }^{\circ} \mathrm{C}$ : (A) Backscattered electron image which shows the presence of a darker secondary phase, (B) EDS spectra of the darker, La-rich phase, (C) EDS spectra of the lighter, La-deficient phase. 


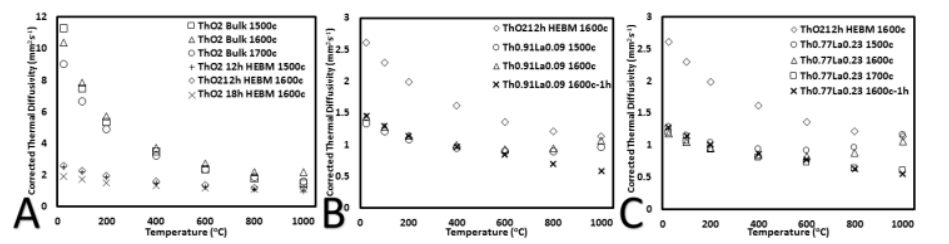

Fig. 10

Thermal diffusivity measurements of

(A) $\mathrm{ThO}_{2}$ (B) $\mathrm{Th}_{0.91} \mathrm{Laa}_{0.09} \mathrm{O}_{1.955}$ (C) $\mathrm{Th}_{0.77} \mathrm{La}_{0.23} \mathrm{O}_{1.885}$. Diffusivity beyond $600{ }^{\circ} \mathrm{C}$ correlates strongly to the presence of the La-deficient phase. 


\section{References}

[1] Sokolov F, Fukuda K, Nawada H. Thorium fuel cycle-potential benefits and challenges. IAEA TECDOC. 2005;1450.

[2] Belle J, Berman R. Thorium dioxide: properties and nuclear applications: USDOE Assistant Secretary for Nuclear Energy, Washington, DC. Office of Naval Reactors; 1984.

[3] Lung M, Gremm O. Perspectives of the thorium fuel cycle. Nuclear Engineering and Design. 1998;180(2):133-46.

[4] Galperin A, Reichert P, Radkowsky A. Thorium fuel for light water reactors-reducing proliferation potential of nuclear power fuel cycle. Science \& Global Security. 1997;6(3):265-90.

[5] Mulford RN, Ellinger F. ThO2-PuO2 and CeO2-PuO2 solid solutions. The Journal of Physical Chemistry. 1958;62(11):1466-7.

[6] Bakker K, Cordfunke E, Konings R, Schram R. Critical evaluation of the thermal properties of Th0 2 and Th $1-y \cup$ y 02 and a survey of the literature data on Th 1-y Pu y 02 . Journal of nuclear materials. 1997;250(1):1-12.

[7] Hubert S, Purans J, Heisbourg G, Moisy P, Dacheux N. Local Structure of Actinide Dioxide Solid Solutions Th1-x U x O2 and Th1-x Pu x 02. Inorganic chemistry. 2006;45(10):3887-94.

[8] Cozzo C, Staicu D, Somers J, Fernandez A, Konings R. Thermal diffusivity and conductivity of thorium-plutonium mixed oxides. Journal of Nuclear Materials. 2011;416(1):135-41.

[9] Aizenshtein M, Shvareva TY, Navrotsky A. Thermochemistry of Lanthana-and Yttria-Doped Thoria. Journal of the American Ceramic Society. 2010;93(12):4142-7.

[10] Murti PS, Mathews C. Thermal diffusivity and thermal conductivity studies on thoriumlanthanum mixed oxide solid solutions. Journal of Physics D: Applied Physics. 1991;24(12):2202. [11] Jain D, Pillai C, Rao B, Kulkarni R, Ramdasan E, Sahoo K. Thermal diffusivity and thermal conductivity of thoria-lanthana solid solutions up to $10 \mathrm{~mol} . \% \mathrm{LaO} 1.5$. Journal of nuclear materials. 2006;353(1):35-41.

[12] Panneerselvam G, Antony M, Vasudevan T. A study on ThO 2-LaO 1.5 solid solution: Lattice thermal expansion measurements and XPS studies. Journal of alloys and compounds. 2006;415(1):26-30. [13] Muta H, Kawano T, Uno M, Ohishi Y, Kurosaki K, Yamanaka S. Lattice parameter and thermal conductivity of Th 1- xMxO 2-y (M=Y, La, Ce, Nd, Gd and U). Journal of Nuclear Materials.

2013;434(1):124-8.

[14] Panneerselvam G, Antony M, Vasudevan T. A study on ThO 2-LaO 1.5 solid solution: synthesis, characterization and estimation of solubility of LaO 1.5 in ThO 2 at $1473 \mathrm{~K}$. Materials Letters. 2004;58(25):3192-6.

[15] Yao T, Scott SM, Xin G, Lian J. TiO 2 doped UO 2 fuels sintered by spark plasma sintering. Journal of Nuclear Materials. 2016;469:251-61.

[16] Furlani E, Aneggi E, de Leitenburg C, Maschio S. High energy ball milling of titania and titaniaceria powder mixtures. Powder Technology. 2014;254:591-6.

[17] Khakpour Z, Youzbashi A, Maghsoudipour A, Ahmadi K. Synthesis of nanosized gadolinium doped ceria solid solution by high energy ball milling. Powder technology. 2011;214(1):117-21.

[18] Lu F, Yao T, Xu J, Wang J, Scott S, Dong Z, et al. Facile low temperature solid state synthesis of iodoapatite by high-energy ball milling. RSC Advances. 2014;4(73):38718-25.

[19] Anselmi-Tamburini U, Garay J, Munir Z, Tacca A, Maglia F, Spinolo G. Spark plasma sintering and characterization of bulk nanostructured fully stabilized zirconia: Part I. Densification studies. Journal of materials research. 2004;19(11):3255-62. 
[20] Munir Z, Anselmi-Tamburini U, Ohyanagi M. The effect of electric field and pressure on the synthesis and consolidation of materials: a review of the spark plasma sintering method. Journal of Materials Science. 2006;41(3):763-77.

[21] TyrpekI V, Cologna M, Robba D, Somers J. Sintering behaviour of nanocrystalline ThO 2 powder using spark plasma sintering. Journal of the European Ceramic Society. 2016;36(3):767-72.

[22] Ge L, Subhash G, Baney RH, Tulenko JS, McKenna E. Densification of uranium dioxide fuel pellets prepared by spark plasma sintering (SPS). Journal of Nuclear Materials. 2013;435(1):1-9.

[23] Choi K, Tong W, Maiani RD, Burkes DE, Munir ZA. Densification of nano-CeO 2 ceramics as nuclear oxide surrogate by spark plasma sintering. Journal of Nuclear Materials. 2010;404(3):210-6.

[24] Shannon R. Revised effective ionic radii and systematic studies of interatomic distances in halides and chalcogenides. Acta Crystallographica Section A. 1976;32(5):751-67.

[25] Tavafoghi Jahromi M, Tan M. Effects of sintering on Y2O3-doped CeO2. Journal of Achievements in Materials and Manufacturing Engineering. 2009;34(2):130-6.

[26] Soyez G, Eastman JA, Thompson LJ, Bai G-R, Baldo PM, McCormick AW, et al. Grain-sizedependent thermal conductivity of nanocrystalline yttria-stabilized zirconia films grown by metalorganic chemical vapor deposition. Applied Physics Letters. 2000;77(8):1155-7. 